\title{
Vertical mixing and coherent anticyclones in the ocean: the role of stratification
}

\author{
I. Koszalka ${ }^{1}$, L. Ceballos ${ }^{2}$, and A. Bracco ${ }^{2}$ \\ ${ }^{1}$ Institute of Geosciences, University of Oslo, 1022 Blinden, 0315 Oslo, Norway \\ ${ }^{2}$ School of Earth and Atmospheric Science, Georgia Institute of Technology, Atlanta, GA 30332, USA
}

Received: 30 September 2009 - Revised: 9 December 2009 - Accepted: 18 December 2009 - Published: 22 January 2010

\begin{abstract}
The role played by wind-forced anticyclones in the vertical transport and mixing at the ocean mesoscale is investigated with a primitive-equation numerical model in an idealized configuration. The focus of this work is to determine how the stratification impacts such transport.

The flows, forced only at the surface by an idealized wind forcing, are predominantly horizontal and, on average, quasigeostrophic. Inside vortex cores and intense filaments, however, the dynamics is strongly ageostrophic.

Mesoscale anticyclones appear as "islands" of increased penetration of wind energy into the ocean interior and they represent the maxima of available potential energy. The amount of available potential energy is directly correlated with the degree of stratification.

The wind energy injected at the surface is transferred at depth through the generation and subsequent straining effect of Vortex Rossby Waves (VRWs), and through nearinertial internal oscillations trapped inside anticyclonic vortices. Both these mechanisms are affected by stratification. Stronger transfer but larger confinement close to the surface is found when the stratification is stronger. For weaker stratification, vertical mixing close to the surface is less intense but below about $150 \mathrm{~m}$ attains substantially higher values due to an increased contribution of both VRWs, whose time scale is on the order of few days, and of near-inertial motions, with a time scale of few hours.
\end{abstract}

\section{Introduction}

The ocean circulation is characterized by a surface wind driven component, a stable stratification below the first few hundred meters of the water column, a meridional over-

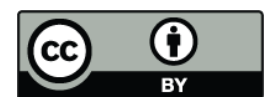

Correspondence to: I. Koszalka

(inga.koszalka@geo.uio.no) turning circulation and turbulent motions. Turbulence in the ocean encompasses many dynamical scales, but at the mesoscale, between 10 and $500 \mathrm{~km}$, the motion is quasi twodimensional and characterized by the presence of coherent vortices. They permeate many oceanic regions and form primarily by internal instability of the flow field along the baroclinically-unstable meanders of boundary currents and jets, locally through the interaction of the flow with the topography, or as a response to the atmospheric forcing that acts as eddy kinetic energy source in oceanic regions far from strong currents (Stammer, 1997; Bracco and Pedlosky, 2003; Spall et al., 2007; Wunsch and Ferrari, 2004).

Eddies are crucial to the local dynamics and have important consequences on tracer dispersion, ocean stirring and mixing processes (Pasquero et al., 2007). Their role on the horizontal motion has been studied extensively in the framework of 2d-barotropic, quasigeostrophic, and shallow water turbulence (e.g., McWilliams and Weiss, 1994; Provenzale, 1999; Bracco et al., 2004; Pasquero et al., 2004). The vertical structure of mesoscale vortices and the associated mixing, however, had long been viewed and parameterized in a simplified manner (Gent and McWilliams, 1995; Griffies, 2000) until recent findings have highlighted their role in transferring wind-forced near-inertial oscillations into the ocean interior (Kunze, 1985; Klein et al., 2004; Danioux et al., 2008). In this respect eddies constitute a different pathway by which inertial energy can reach the ocean interior and their contribution to vertical mixing may be fundamental in maintaining the meridional overturning circulation. In particular, cyclonic and anticyclonic eddies expel or trap, respectively, inertial oscillations as shown by Kunze (1985) for quasigeostrophic flows and further investigated by Danioux et al. (2008) in a primitive-equation setup. Following these works, Koszalka et al. (2009) have shown that in a strongly stratified ocean the vertical circulation associated with windforced anticyclones is one order of magnitude more intense than predicted by the quasigeostrophic omega equation and

Published by Copernicus Publications on behalf of the European Geosciences Union and the American Geophysical Union. 

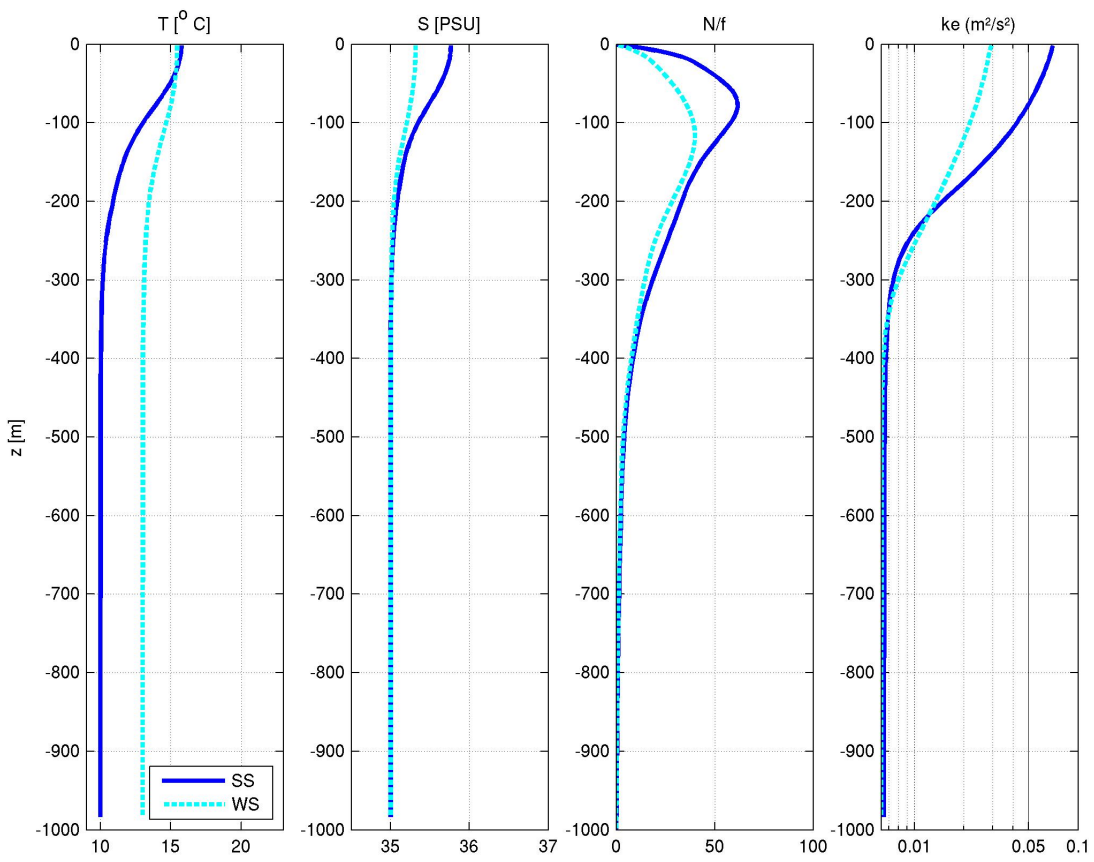

Fig. 1. Domain-averaged profiles in the two configurations WS and SS, time-averaged over 100 days. From left to right: temperature ( $T$ ), salinity $(S)$, Brunt-Vaisala frequency $(N / f)$, and kinetic energy (ke).

exhibits a complex spatial pattern, akin to the one reported for frontal regions. At local scales, the strong and complex vertical mixing associated with the eddies may help interpreting recent observations of biological fields (Benitez-Nelson et al., 2007; McGillicuddy et al., 2007).

Here we use the idealized set-up of Koszalka et al. (2009) and high vertical resolution to investigate with a primitive equation model how stratification impacts the representation of vertical transport and mixing with a wind-driven mesoscale eddy field. The horizontal and vertical extension of oceanic eddies is affected by the local stratification (Smith and Vallis, 2001, 2002; Arbic et al., 2007). However, while it has been shown that the ambient stratification does not affect the horizontal transport and mixing properties of mesoscale vortices (Bracco et al., 2004), its influence in setting the vertical ones has not been investigated in details.

In light of the recent findings on wind-forced near-inertial oscillations, we have chosen a flow configuration that insures a predominance of anticyclones. Dominance of anticyclones has been found in a number of numerical and theoretical solutions of the geostrophic turbulence that are representative of the ocean interior (Polvani et al., 1994; Arai and Yamagata, 1994; Cho and Polvani, 1996; Yavneh et al., 1997). Here we consider two different vertical stratification profiles and we analyze the eddy population that emerges in an idealized, wind-forced portion of the open ocean. We study the transfer of the wind energy into the underlying flow and its redistribution in the fluid column, focusing on the vertical transport properties of the mesoscale vortices.

\section{Numerical model}

In this study we use the Regional Ocean Modelling System (ROMS). ROMS is an incompressible, free-surface, hydrostatic, primitive-equations, circulation model (Shchepetkin and McWilliams, 2005) with a generalized vertical, terrainfollowing, coordinate system (s-coordinate). The idealized configuration implemented is similar to the one described in Koszalka et al. (2009). The domain is doubly periodic, with constant depth $H=1000 \mathrm{~m}$ and lateral size $L=256 \mathrm{~km}$. The resolution is $\Delta x=1 \mathrm{~km}$ in the horizontal and 80 layers in the vertical, 23 of which confined in the upper $100 \mathrm{~m}$ of the water column. The Coriolis frequency is $f=10^{-4} \mathrm{~s}^{-1}$.

In the ocean, the degree of stratification is described by the buoyancy frequency, $N(z)=\left(-\frac{g}{\rho} \frac{d \rho}{d z}\right)$, where $z$ is a local vertical coordinate and $\rho$ is potential density. In most oceanic regions $N / f$, which measures the relative importance of stratification and rotation, is greater than 1 , and is typically of order 10 or more, with larger values attained near the surface than at depth.

Here we consider two vertical stratifications, as shown in Fig. 1. We do not apply a relaxation to initially imposed salinity and temperature profiles, and this allows us to study the effect of the presence of the vortices on the stratification. Figure 1 refers to the mean state calculated over a 100day period on which we focus the analysis, and the departure from it is briefly discussed in Sect. 3.1 (see Fig. 5c). In the following we refer to the strongest stratified case as SS and to the weaker one as WS. The SS profile is similar to the one 
in Koszalka et al. (2009) but for the absence of the mixed layer, and is representative of extratropical open ocean waters. As discussed in Koszalka et al. (2009) a mixed layer would slightly reduce the vertical velocities below the surface, limiting the transfer of momentum to deeper waters, but it does not modify the general structure of the vertical velocity field. The first internal Rossby radius of deformation, given by $L_{\mathrm{I}}=\frac{\sqrt{<N>H}}{f}$ (Vallis, 2006) is $L_{\mathrm{I}}^{\mathrm{SS}} \approx 15 \mathrm{~km}$ and $L_{\mathrm{I}}^{\mathrm{WS}} \approx 11 \mathrm{~km}^{1}$. The (barotropic) Rossby radius of deformation is $L_{o}=\sqrt{g H} / f \approx 1000 \mathrm{~km}$ in both configurations.

Biharmonic horizontal diffusion acts with coefficient $A_{\mathrm{H}}=10^{6} \mathrm{~m}^{4} \mathrm{~s}^{-1}$ on all fields and runs, and the nondimensional quadratic bottom drag parameter is $3 \times 10^{-4}$. A non-local K-Profile Parameterization (KPP) scheme (Large et al., 1994) is used to parameterize vertical mixing. The scheme modifies the background value of vertical mixing coefficient, $K v_{\text {coef }}=10^{-6} \mathrm{~m}^{2} \mathrm{~s}^{-1}$ in both runs, in the surface boundary layer by employing the Monin-Obukhov similarity theory, and in the interior by accounting for contributions due to shear instability as function of the local Richardson number and to internal wave breaking assumed to be inversely proportional to $N$. The vertical dissipation is thus function of the system state and will vary between the configurations.

The fluid is initially at rest and it is constantly forced by a spatially-variable, narrow-band sinusoidal wind stress of amplitude $0.1 \mathrm{~N} \mathrm{~m}^{-2}$, centered on the wavenumber $k_{x}=k_{y}=3$, i.e. with a radial wavenumber $k_{\mathrm{f}}=\sqrt{k_{x}^{2}+k_{y}^{2}} \approx 4$ and length scale $L_{\mathrm{f}} \approx 64 \mathrm{~km}$. This idealized forcing is used as an artifice to maintain the mesoscale turbulence in the homogeneous domain in lieu of the inclusion of inhomogeneous mean-flow instability processes. It was verified in Koszalka et al. (2009) that the evolution and transport properties of the simulated flow do not depend critically on the detail of the forcing field for a given radial wavenumber. The wind forcing is identical in all configurations. Radiative forcing is not included in these idealized runs.

\section{Results}

\subsection{Wind-forced vortices}

Within the time scale of the inertial period, the wind forcing sets up a circulation in the Ekman layer with depth approximately given by $d_{\mathrm{E}}=\frac{2<K v\rangle}{f}$ (Vallis, 2006), where $\langle K v\rangle$ is the surface-averaged vertical mixing coefficient for momentum in the KPP scheme. In all runs $d_{\mathrm{E}}$ is about $6 \mathrm{~m}$.

After about 200 days, the system reaches a statistical stationary state, on which we focus our analysis. The corresponding domain-averaged profiles of temperature, salin-

\footnotetext{
${ }^{1}$ We follow Vallis (2006) and use the definition without inclusion of the factor of $\pi$. The estimates given by the normal mode analysis (dynmodes.m of J. Klinck) give slightly higher values, $L_{\mathrm{I}}^{S S} \approx 20 \mathrm{~km}$ and $L_{\mathrm{I}}^{\mathrm{WS}} \approx 15 \mathrm{~km}$.
}

ity, Brunt-Vaisala frequency and kinetic energy are shown in Fig. 1. The dynamics is surface-intensified in both cases due to the chosen forcing. The stratification influences depth and strength of the energy transfers and this results in the nearsurface flow in SS being twice as strong as in WS case and slightly weaker at mid-depth.

The flow is dominated by the presence of coherent vortices, predominantly anticyclonic, as shown by the vertical component of the relative vorticity, $\zeta=\partial_{x} v-\partial_{y} u$, in Fig. 2a-b. The vortices are long-lived (up to several months) and undergo complex evolution involving perturbations under the straining field due to wind forcing, instabilities in the vortex cores and merging events.

The asymmetry in the vortex population in stratified flows with small $L_{\mathrm{I}}$ and finite $R o$ has been discussed in Koszalka et al. (2009) and is linked to the straining field exerted by vortex Rossby Waves (VRWs), which are evident in the sub-surface vertical velocity $(w)$ field (Fig. $2 \mathrm{c}-\mathrm{d}$; the $w$ field is described at more length in Sect. 3.3). As shown by Graves et al. (2006) with a weakly-nonlinear shallowwater model, in the vortex relaxation process VRWs lead to a substantial weakening of cyclones and a strengthening and re-axisymmetrization of perturbed anticyclones. The asymmetry, quantified by the skewness of vorticity distributions (Fig. 3a), is most pronounced at depth, and is compensated by the presence of strong cyclonic filaments close to the surface. The depth of maximum asymmetry is linked to the penetration of the vortex cores, in turn determined by the stratification, and is approximately $\approx 200 \mathrm{~m}$ and $300 \mathrm{~m}$ in SS and WS respectively (Fig. 2e-f), consistent with the vertical distribution of kinetic energy (see Fig. 1).

The average Rossby number, $\langle R o\rangle=\frac{U}{f L_{\mathrm{I}}}$, is $\approx 0.2$ for both configurations, independent on stratification as the difference in the intensity of the flow measured by $U$ is compensated by the difference in $L_{\mathrm{I}}$. The local value of $R o$, estimated by $\zeta / f$, however, exceeds 2 in the vortex cores and filaments.

The r.m.s. (root mean square) horizontal velocity exceeds the r.m.s. of the vertical velocity by at least three orders of magnitude, and the horizontal divergence, $\chi=\partial_{x} u+\partial_{y} v$, is at least one order of magnitude smaller than relative vorticity throughout the fluid column (not shown). We can therefore conclude that the motion is primarily horizontal and, on average, quasigeostrophic.

Vertical profiles of area-averaged ratio of the baroclinic (depth-dependent) component of the horizontal kinetic energy, $U_{\mathrm{BRCL}}$, to the barotropic (depth-independent) one, $U_{\mathrm{BAR}}$, are shown in Fig. $3 \mathrm{~b}$. At the surface the baroclinic contribution overcomes the barotropic one by 1.7 times in the SS run, while it is of comparable importance in the WS case. The baroclinic contribution decreases with depth, reaching its minimum in correspondence of the average penetration depth of the vortex cores. Below the vortices the two contributions remain constant, at a value slightly higher in the SS case. Qualitatively analogous curves are obtained selecting only regions of the flow within and around the vortices 

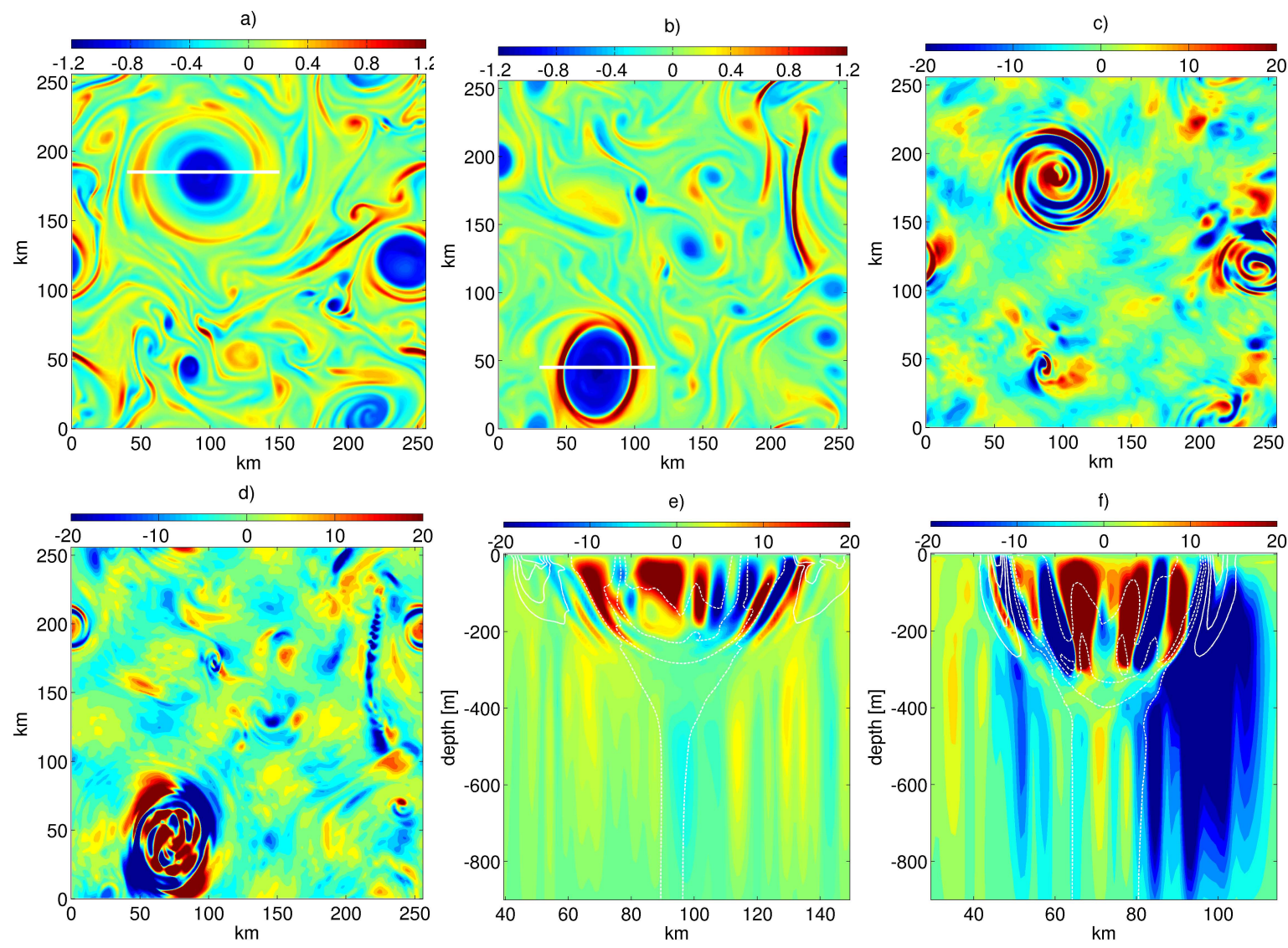

Fig. 2. Instantaneous snapshots of relative vorticity at surface (10-m depth) scaled by the Coriolis parameter, $\zeta / f$ for (a) SS and (b) WS case. Instantenous vertical velocity field $w\left(\mathrm{~m} \mathrm{day}^{-1}\right)$ at $80 \mathrm{~m}$ depth in (c) SS and (d) WS runs. Instantaneous vertical sections of $w\left(\mathrm{~m}\right.$ day $\left.{ }^{-1}\right)$ through vortices in (e) SS and (f) WS, respectively.

and in the strongest filaments, with ratios of $U_{\mathrm{BRCL}} / U_{\mathrm{BAR}}$ of 2.0 and 1.3 at the surface for SS and WS runs, respectively (not shown).

The wavenumber spectra of the barotropic and baroclinic components of the horizontal kinetic energy are separately shown in Fig. 4a-b. Both barotropic energy spectra exhibit slopes significantly steeper than $k^{-3}$ for $k_{f}<k<4 \mathrm{~km}$, i.e. in the direct energy cascade range (below $4 \mathrm{~km}$ horizontal diffusion dominates the spectral slopes). The inverse energy cascade is inhibited in our runs featuring finite $L_{\mathrm{I}}$ regime, being the internal Rossby radius $L_{\mathrm{I}}$ smaller than the forcing scale (Larichev and McWilliams, 1991).

The wavenumber spectra of the baroclinic component in SS and WS (Fig. 4b) have slopes slightly steeper than $k^{-3}$ for $k>k_{f}$ in the upper few hundred meters, consistent with the behaviour of 2-D turbulence and stratified quasigeostrophic turbulence in the direct enstrophy cascade (Babiano and Provenzale, 2007; Smith and Vallis, 2002). Note that the higher amount of surface baroclinic energy found in the SS case (Fig. 3b) appears to be concentrated at scales larger than the forcing; the $W S$ configuration is characterized by smaller $L_{\mathrm{I}}$ and thus stronger inhibition of the transfers in the inverse energy cascade range.

Recently Arbic et al. (2007) considered a two-layer quasigeostrophic model to investigate the dynamics of Ekmandamped, turbulent oceanic flows with surface-intensified stratification, and found that the baroclinic energy is spatially localized at horizontal scales near $L_{\mathrm{I}}$ while the barotropic energy dominates the spatial average, in qualitatively agreement with the results presented here.

To quantify how mesoscale vortices and submesoscale filaments affect vertical transport in the ocean we first analyze the conversion of energy injected at the surface by the idealized wind. The transfer of wind energy into the ocean can be quantified by the wind power, i.e. the mean rate of the wind work, given by the mean product of the wind stress and the surface velocity, $\mathrm{WW}=\iint\langle\tau u\rangle d x d y$ (e.g. Wunsch and Ferrari, 2004; Brown and Fedorov, 2008). In both runs the mean wind power per unit area WW' is 
a)

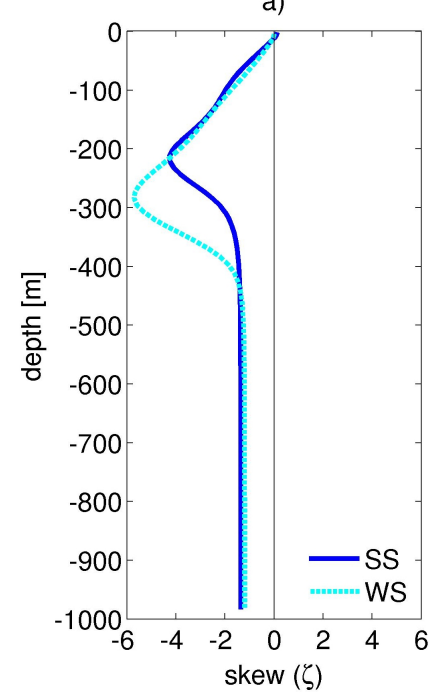

b)

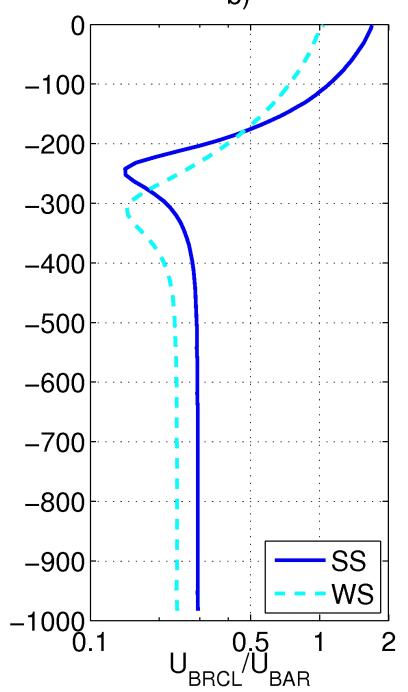

Fig. 3. Surface-averaged vertical profiles: (a) skewness of the vertical component of the relative vorticity $\zeta$, (b) ratio of baroclinic (depth-dependent) to barotropic component of the horizontal kinetic energy. All statistical measures are averaged over a time period of 100 days.

approximately $0.001 \mathrm{~W} / \mathrm{m}^{2}$, as an identical wind stress forces the circulation (Fig. 5a). Such a value is representative of vast regions of the ocean (Brown and Fedorov, 2008). Due to stronger surface circulation in the SS case, however, the standard deviation of the two time series differs quite significantly $\left(7 \times 10^{-3} \mathrm{~W} / \mathrm{m}^{2}\right.$ for SS and $4 \times 10^{-3} \mathrm{~W} / \mathrm{m}^{2}$ for WS). The low frequency variability in the time series is associated with the development of the large vortices that dominate the overall dynamics (Fig. 7b).

Only a portion of the energy introduced by the wind is dynamically active and can be transformed in kinetic energy via baroclinic instability. This is the available potential energy of the system (in Joules), defined as:

$\mathrm{APE}=1 / 2 \iiint\left\langle\hat{\rho}^{2} / \gamma^{2}\right\rangle d x d y d z$,

where $\gamma^{2}=\left|(1 / g)\left(d \rho^{*} / d z\right)\right|, \rho^{*}(z)$ represents the density profile averaged over the 100-day period and over the horizontal domain and $\hat{\rho}(x, y, z, t)$ is the deviation from $\rho^{*}$ (Oort et al., 1989; Brown and Fedorov, 2008). The domainintegrated APE differs quite significantly between the two runs, being in the SS case twice as strong as in WS case $\left(16 \times 10^{9} \mathrm{~J}\right.$ vs. $8.5 \times 10^{9} \mathrm{~J}$, respectively). The vertical structure of the integrand averaged over the 100 days is shown in Fig. 5b, and is reminiscent of the eddy APE structure found in Oort et al. (1989). It decreases rapidly in the subsurface within the depth of the eddy penetration (Fig. 3a). This subsurface reduction in APE occurs when the dynamics act to flatten the isopycnals (Fig. 5c), as during conversion of APE into kinetic energy in baroclinic instability processes (Vallis,
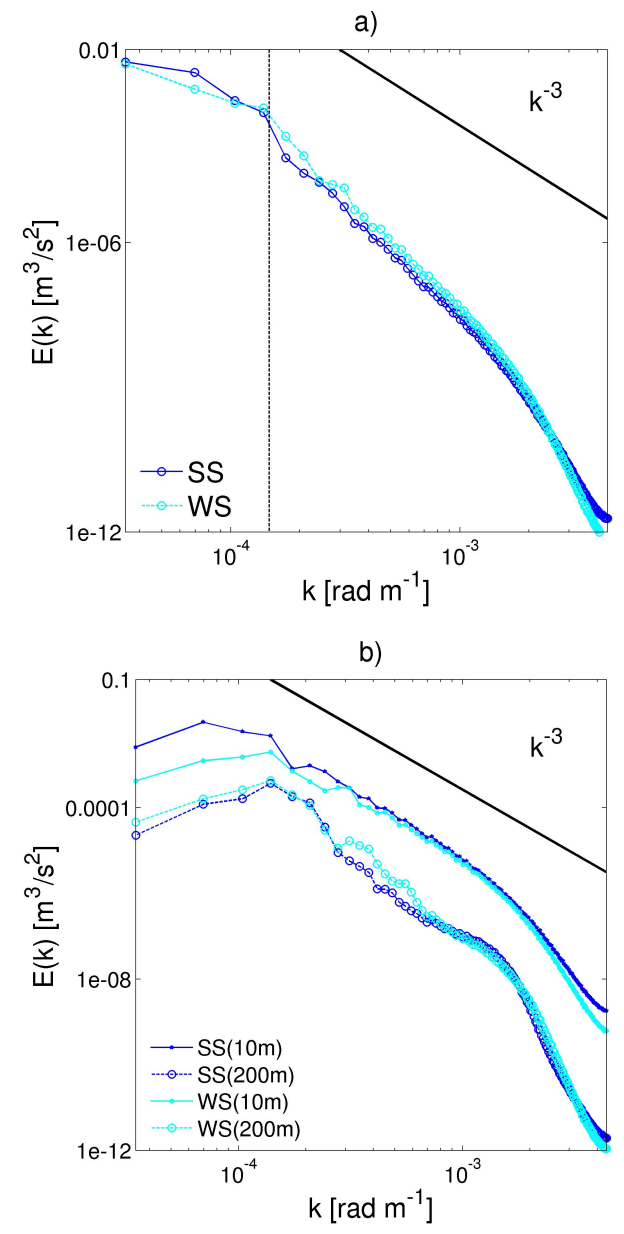

Fig. 4. Top: horizontal wavenumber spectra of the horizontal kinetic energy, time-averaged over 100 days: (a) barotropic (depth-averaged) component. The black line indicates the forcing wavenumber, (b) baroclinic (depth-dependent) component.

2006). The vortices correspond to local maxima in verticallyintegrated APE (not shown). They cover only $5 \%$ of the domain area in both SS and WS runs, but they account for about $65 \%$ of the total vertically-integrated APE in both cases. Thus, given the different levels of APE, the role of vortices in the energy conversion increases with increasing strength of the stratification resulting in higher levels of the baroclinic energy in the near-surface layer (Fig. 3b). A vertical section of the integrand of the APE through an eddy is shown in Fig. 5d. The surface input of APE coincides with the position of the vortex, where both the wind energy input and the deviation for the average density are strongest. The depletion in APE associated with conversion to the kinetic energy occurs in the subsurface "inside" this mesoscale coherent structure. $^{2}$

\footnotetext{
${ }^{2}$ To quantify the area covered by the vortices we used the
} 

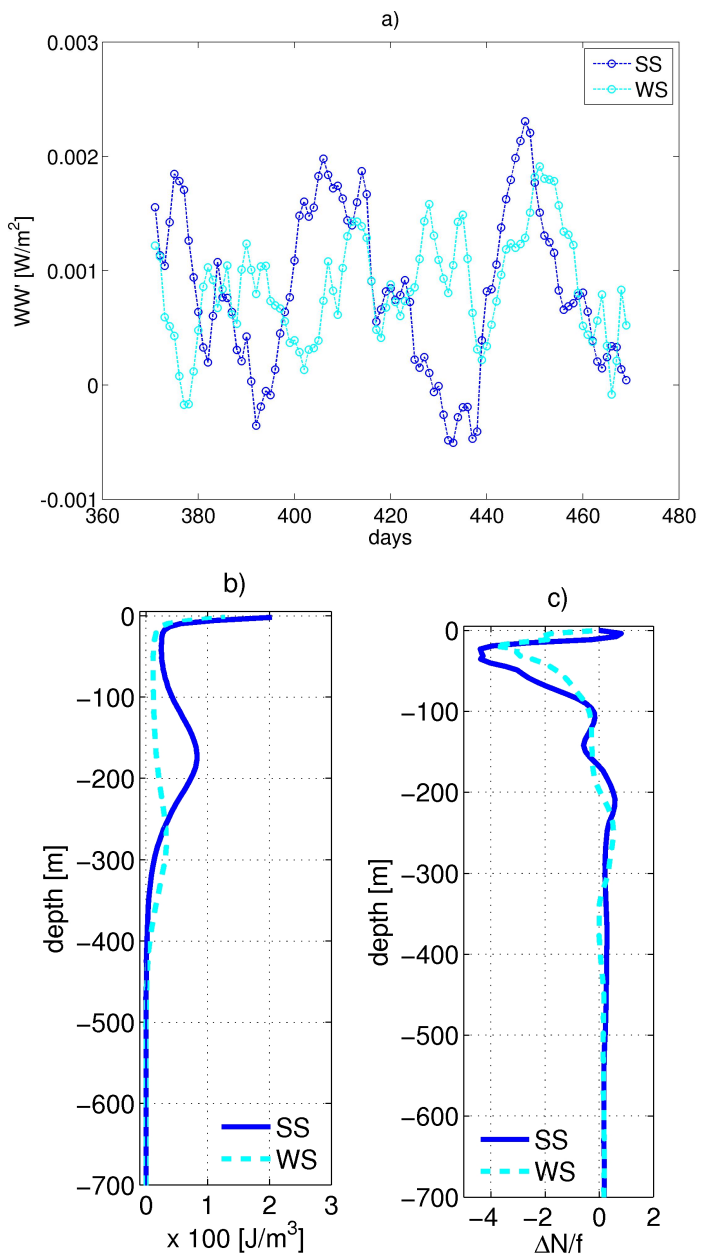

d)

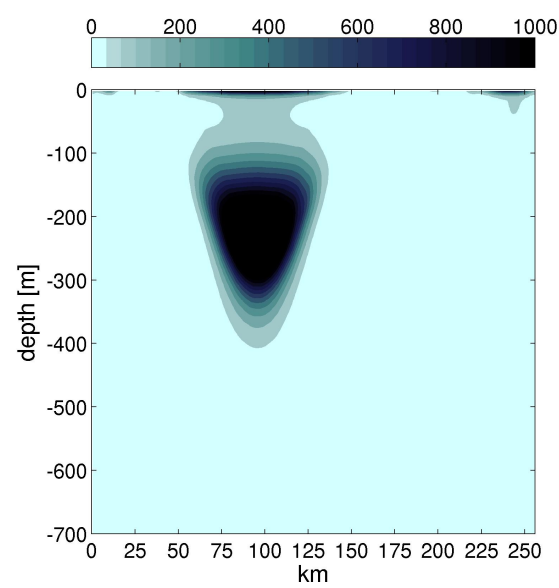

Fig. 5. (a) Time series of the mean wind power per unit area WW' in $\left(\mathrm{W} \mathrm{m}^{-2}\right)$. (b) Vertical profile of the integrand of the mean APE, averaged on the horizontal domain and over 100 days. (c) Vertical profile of the area-averaged difference in stratification $\Delta N=$ $N\left(t_{100}\right)-N\left(t_{o}\right)$ over the 100-day period upon which the analysis is focused, scaled with the Coriolis parameter $f$. (d) A snapshot of the vertical section of the integrand in Eq. (1) (APE) for the SS case encompassing the section through the vortex shown in Fig. 2 a.
The presence of vortices and filaments may further affect the vertical transport in two ways: by modifying the vertical mixing coefficient used in the KPP parameterization scheme, and by inducing a vertical circulation. These two mechanisms are separately investigated below.

\subsection{Vertical mixing due to the presence of the vortices}

The vertical profiles of the horizontally averaged effective vertical mixing term $K v$ determined by the KPP scheme (Large et al., 1994) are shown in Fig. 6a. The mean value of $K v$ does not vary significantly with stratification, being modestly weaker between 100 and $200 \mathrm{~m}$ in the SS case, and features a similar vertical structure in the SS and WS cases. The constant background value of $K v_{\text {coef }}$ is magnified in the surface boundary layer which is estimated by the KPP scheme to be $d_{b} \approx 30 \mathrm{~m}$ and $d_{b} \approx 40 \mathrm{~m}$ for SS and WS runs, respectively. Figure $6 \mathrm{~b}$ shows a horizontal slice of $K v$ at $30 \mathrm{~m}$ for the SS run. Near the surface, due to locally strong vertical shear and reduced stratification, the anticyclonic vortices act like "islands" of enhanced vertical mixing, and thus enhanced transfer of momentum into deeper layers. The value of $K v$, that is inversely proportional to the ambient value of $N$, increases again below $300 \mathrm{~m}$, where $N$ is small (Fig. 1) and conditions are favorable to internal-wave breaking phenomena that the scheme aims at parameterizing (Large et al., 1994).

\subsection{Vertical circulation}

A snapshot of the instantaneous vertical velocity field at $80 \mathrm{~m}$ is shown in Fig. 2c-d for the SS and WS cases, respectively. Vertical velocities are the most intense in the vicinity of the vortices and reveal a complex small scale structure. The vertical velocity variance profiles (averaged over 100 days, Fig. 7a) reveal that the maximum of $w$ increases with the magnitude of the stratification. The SS case exhibits the strongest vertical velocities close to the surface with a maximum at about $60 \mathrm{~m}$. This is related to an enhancement of the momentum transfer within the vortex cores that are constrained in the first few hundred meters, as inferred from the horizontal kinetic energy spectra. Few vortices can penetrate deeper and induce high vertical velocities as deep as $600 \mathrm{~m}$. For weaker stratification we find smaller vertical velocities close to the surface and a shift of the maximum to deeper layers, due to the increased energy transfer in the vertical. Below this maximum, however, the WS case displays higher velocities than SS through the remaining of the water column, with a core of high values localized between 100

Okubo-Weiss parameter $\mathrm{OW}=S^{2}-\zeta^{2}$, where $S^{2}$ is squared strain, and $\zeta$ is vorticity (Weiss, 1981). OW $<-\sigma_{\text {ow }}$ identifies vortex cores and $\mathrm{OW}>\sigma_{\mathrm{ow}}$ characterizes the cyclonic rings around the vortices and the strongest filaments. For a discussion on the use of $\mathrm{OW}$ in QG and PE flows see (Petersen et al., 2006; Koszalka et al., 2009). 


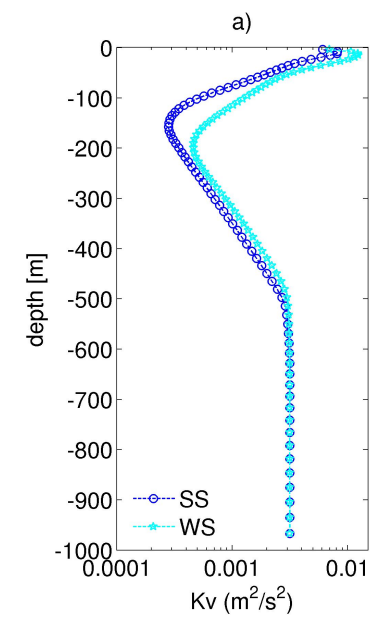

b)

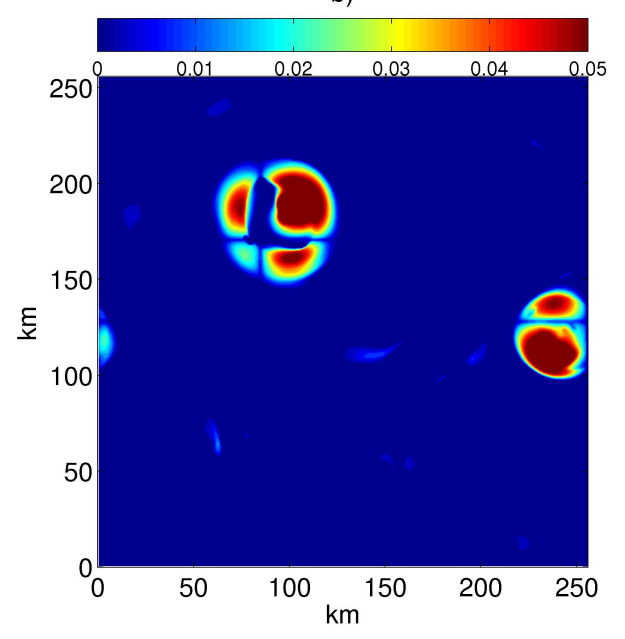

Fig. 6. (a) Area-averaged profiles of the vertical mixing coefficent for momentum for the two simulations, averaged over 100 days. (b) A snapshot of the vertical mixing coefficient at 30-m depth for the SS case in $\mathrm{m}^{2} \mathrm{~s}^{-2}$.

and $500 \mathrm{~m}$ of depth. The overall structure of the velocity field variance displays significant differences in the two cases, despite a relatively small change in stratification.

The overall vertical structure of the vertical velocity field, including the exact positions of the depth maxima, features a low-frequency variability strongly linked to the dynamics of the anticyclonic vortices dominating the flow, as indicated by the Hovmöller diagrams of velocity variance and relative vorticity anomalies, shown for the SS case in Fig. 7b.

The frequency spectra of $w$ (Fig. 7c) reveal the presence of near-inertial internal waves in the deeper layers, broadly in correspondence to the position of the vertical velocity maxima in Fig. 7a. The near-inertial peak is shifted towards superinertial frequencies. This results from the polarization of near-inertial oscillations by the eddy relative vorticity that shifts the phase of the oscillations by $-\zeta t / 2$. Such a shift, in turn, affects the dispersion properties of the waves and induces a growth of their amplitude ("trapping") within anticyclonic eddies, and a decrease in cyclonic regions (Kunze, 1985; Danioux et al., 2008). The reduction of the horizontal scales of the waves due to their dispersion leads also to increased energy dissipation via scale-selective horizontal mixing.

Anticyclonic vortices are also associated with an increased vertical transfer of momentum to deeper layers, and with the emergence of a maximum in $\sigma_{w}$ at depth, in agreement with the analytical and numerical investigation of this "chimney effect of eddies" by Danioux et al. (2008). In their study, focused on an unstable jet forced by time-varying but spatiallyuniform winds, the deep maximum was captured by the lowest vertical normal modes. They find that the signature of near inertial motions related to the first mode is a peak in the frequency spectra close to twice the inertial frequency $2 f_{\mathrm{p}}$. This is consistent with our results for both SS and WS integrations, but for broader peaks due to the dispersion of the waves. The emergence of this scale has been explained analytically using a shallow-water model in Danioux and Klein (2008) as a local resonance in the wavenumber space that follows the excitation by the eddy relative vorticity and involves the nonlinear term $v \partial_{y} h$, where $h$ is the free-surface. In our fully 3-D primitive-equation model, this translates into $\left(u \partial_{x} w, v \partial_{y} w\right)$ terms in the momentum equation and corresponds to the "tilting term" in Eq. (2) below.

Near the surface the vertical velocity is dominated by low(er) frequency motions. Vortices at different scale set the spectral slope and the overall behavior of the vertical velocities. Their contribution is modulated by the presence of vortex Rossby Waves that imprint their signature at subinertial time scales with a broad peak centered at $\approx 4$ day. This time scale is consistent with the prediction obtained from the dispersion relation used in Koszalka et al. (2009) for $L_{\mathrm{I}} \approx 11-15 \mathrm{~km}$ as in our simulations. The contribution of the frequencies in the VRW-range is higher in the WS run compared to the $S S$ run as the importance of VRWs in the evolution of vortices increases with decreasing $L_{\mathrm{I}}$ (Graves et al., 2006; Koszalka et al., 2009), leading to larger degree of cyclone-anticyclone asymmetry (Fig. 3a).

At $200 \mathrm{~m}$ and $600 \mathrm{~m}$ depth the contributions of nearinertial motions and VRWs are significantly more important in the WS case, suggesting that mixing below the surface is strongly enhanced for reduced stratification through those two mechanisms. Near-inertial waves trapped within anticyclones remain stronger in the WS case at all depths (see Fig. 2e-f).

The physical processes contributing to the vertical velocity field, VRWs close to the surface, and near-inertial waves below the first $100 \mathrm{~m}$, can be also identified considering the $w$ diagnostic equation derived in Koszalka et al. (2009) and 
a)

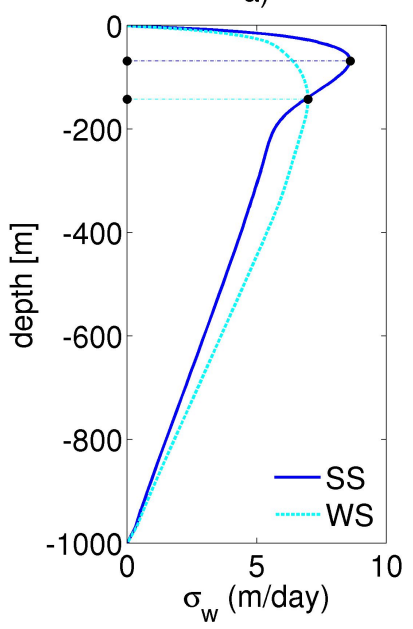

b)
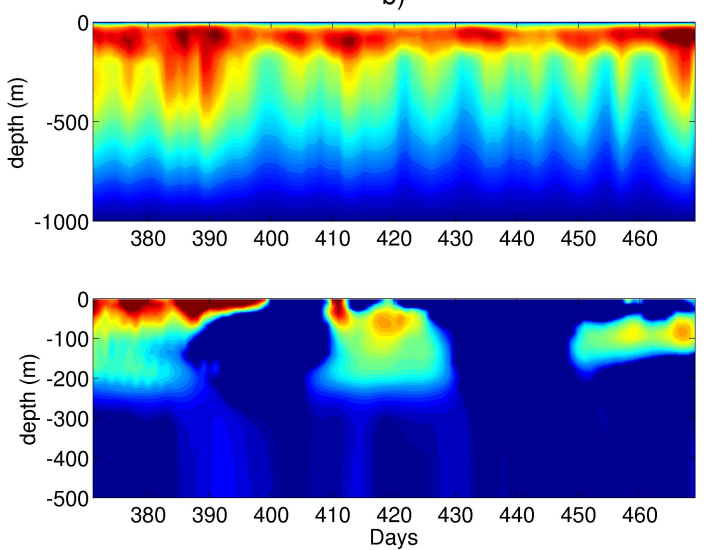

c)

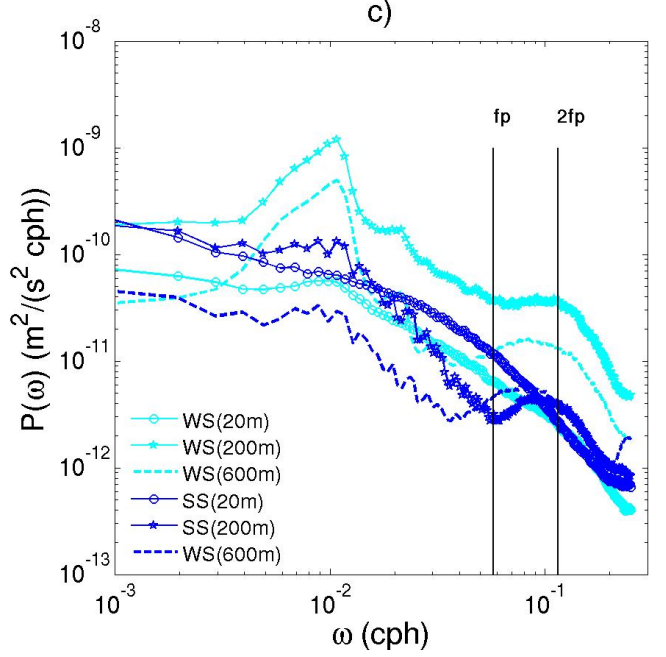

Fig. 7. (a) Area-averaged vertical profiles of $\sigma_{w}$ for varying degree of stratification, averaged over 100 days. The position of the maxima are marked by dashed lines. (b) Hovmöller diagrams of area-averaged profiles of the vertical velocity (top) and deviation from the mean r.m.s of relative vorticity (bottom) for the SS case ( $d t=1$ day). (c) Frequency spectra of the vertical velocity at $20 \mathrm{~m}$, $200 \mathrm{~m}$, and $600 \mathrm{~m}$, with vertical lines marking the inertial frequency $\left(f_{p}\right)$ and twice the inertial frequency $\left(2 f_{p}\right)$. here repeated (see their Appendix for full derivation):

$$
\begin{aligned}
& \text { FREE SURFACE } \\
& w(x, y, z)=\frac{D \eta}{D t} \\
& \text { ADVECTIVE } \\
& -\int_{z}^{\eta} \alpha_{1}\left[\frac{\partial \zeta_{1}}{\partial t}+u \frac{\partial \zeta_{1}}{\partial x}+v \frac{\partial \zeta_{1}}{\partial y}+w \frac{\partial \zeta_{1}}{\partial z}\right] d z \\
& \text { ADVECTIVE } \\
& -\int_{z}^{\eta} \alpha_{2}\left[\frac{\partial \zeta_{2}}{\partial t}+u \frac{\partial \zeta_{2}}{\partial x}+v \frac{\partial \zeta_{2}}{\partial y}+w \frac{\partial \zeta_{2}}{\partial z}\right] d z \\
& \text { STRETCHING } \\
& -\int_{z}^{\eta} \alpha_{1}\left[\chi_{2} \zeta_{1}\right] d z-\int_{z}^{\eta} \alpha_{2}\left[\chi_{1} \zeta_{2}\right] d z \\
& \text { TILTING } \\
& -\int_{z}^{\eta} \alpha_{1}\left[\frac{\partial w}{\partial x} \frac{\partial v}{\partial z}\right] d z+\int_{z}^{\eta} \alpha_{2}\left[\frac{\partial w}{\partial y} \frac{\partial u}{\partial z}\right] d z \\
& \text { WIND STRESS } \\
& +\int_{z}^{\eta} \frac{\alpha_{2}}{\rho_{o}}\left[-\frac{\partial}{\partial y} \frac{\partial \tau_{x}}{\partial z}\right] d z \\
& \text { HORIZONTAL MIXING } \\
& +\sum_{i=1,2} \int_{z}^{\eta} \alpha_{i} A_{H}\left(\frac{\partial^{4} \zeta_{i}}{\partial x^{4}}+\frac{\partial^{4} \zeta_{i}}{\partial y^{4}}\right) d z \\
& \text { VERTICAL MIXING } \\
& +\int_{z}^{\eta} \alpha_{1} \frac{\partial}{\partial x} \frac{\partial}{\partial z}\left(K_{v} \frac{\partial v}{\partial z}\right) d z-\int_{z}^{\eta} \alpha_{2} \frac{\partial}{\partial y} \frac{\partial}{\partial z}\left(K_{v} \frac{\partial u}{\partial z}\right) d z,
\end{aligned}
$$

where $\zeta_{1}=\partial v / \partial x, \zeta_{2}=-\partial u / \partial y, \chi_{1}=\partial u / \partial x, \chi_{2}=\partial v / \partial y$, $\alpha_{1}=\left(f+\zeta_{1}\right)^{-1}$ and $\alpha_{2}=\left(f+\zeta_{2}\right)^{-1}$.

In both runs the nonlinear advective (or ageostrophic) and stretching terms dominate. These two terms appear in the form of azimuthal wavenumber $k_{\mathrm{A}}=4$ disturbances around the vortex cores, are strongly anticorrelated and largely cancel each other (see Koszalka et al. (2009) and their Fig. 4). As a result, the tilting term dominates the overall pattern of $w$ near the vortex cores in the upper $150 \mathrm{~m}$. The amplitude of the vertical velocity field, however, is dominated by the regions where the nonlinear advective (or ageostrophic) and stretching terms do not cancel out. These three dominant contributions when averaged over the inertial period bear clearly expression of the VRWs, characterized by a subinertial time scales of 3-5 days (Graves et al., 2006; Koszalka et al., 2009). In the upper $100 \mathrm{~m}$ the contribution of the vertical mixing term is also significant and reaches $10 \mathrm{~m} / \mathrm{s}$, with a spatial variability clearly related to the presence of the vortices (Koszalka et al., 2009).

The vertical profiles of the four largest contributions in Eq. (2) are shown in Fig. 8a for the SS case and for the upper $500 \mathrm{~m}$ of the water column. Results are similar for the WS integration. The vertical mixing and tilting terms, related to wave motions, are at least one magnitude lower than the advection and stretching terms related to the evolution of the vertical component of the relative vorticity and determined 
a)

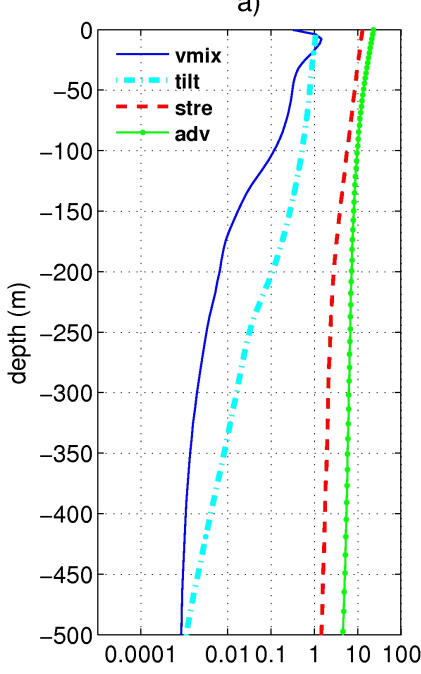

b)

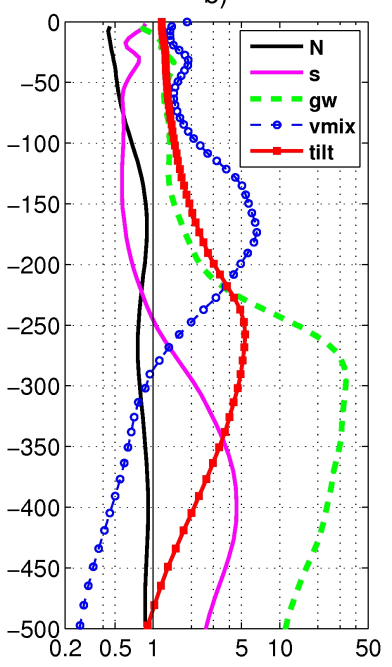

Fig. 8. (a) Variance of one-day averaged contributions in Eq. 2 of the four largest terms in the SS case in $\mathrm{m} / \mathrm{s}$. (b) WS over SS ratios of Brunt-Vaisala frequency $(N)$, of the magnitude of vertical shear $\sqrt{\left(\partial_{z} u\right)^{2}+\left(\partial_{z} v\right)^{2}}(s)$, of the magnitude of the horizontal gradient of $w, \sqrt{\left(\partial_{x} w\right)^{2}+\left(\partial_{y} w\right)^{2}}$ (gw), and of vertical mixing (vmix) and tilting (tilt) terms.

mainly by the horizontal dynamics ${ }^{3}$. To further illustrate the physical processes responsible for the differences in the vertical mixing and tilting contributions at mid-depth in the two runs, we plot the ratio of the vertical mixing and tilting terms obtained for WS and SS, together with ratios of the BruntVaisala frequency, and of the relative magnitude of the vertical shear and of the horizontal gradient of $w$ (Fig. 8b). The vertical mixing term is up to 5 times larger in the WS case in a depth range of 100-300 $\mathrm{m}$, due to the differences in both the profile of $K v$ (Fig. 6a) and in stratification. The magnitude of the tilting term, on the other hand, appears to be controlled by the horizontal gradients of $w$ that are expression of small-scale internal oscillations and are more energetic in the WS case as seen in the frequency spectra (Fig. 7c) .

\section{Discussion and conclusions}

The maintenance of the ocean circulation requires energy input from the atmospheric wind field, and the wind forcing is believed to be the main driving force for the mesoscale eddies far from the ocean boundaries (Stammer, 1997). Winds acting on the sea surface produce direct conversion of atmospheric kinetic energy into oceanic kinetic and potential ener-

\footnotetext{
${ }^{3}$ The contribution of horizontal advection in the ADVECTIVE term in Eq. (2) is at least one order of magnitude larger than the contribution of vertical advection throughout the fluid column.
}

gies. A fraction of this input feeds into the large-scale general circulation, partly removed by baroclinic instability whereby the mesoscale flow structures arise. However the pathways of its further transmission to increasingly small scales in the ocean interior, and the role of mesoscale circulation in this transmission, are still uncertain (Wunsch and Ferrari, 2004; Brown and Fedorov, 2008). Near inertial motions are the most obvious candidate for small-scale mixing in the ocean, with various possible generation mechanisms.

Recent works point to mesoscale vortices as responsible for transferring wind energy into the ocean interior through near inertial oscillations (Kunze, 1985; Klein et al., 2004; Danioux et al., 2008). Here, with a primitive-equation numerical model in an idealized set-up, we investigated the role of wind-forced anticyclones in the vertical mixing and the dependence of the (modeled) mesoscale ocean circulation on stratification, considering two different stratification profiles.

The flows are forced by an identical wind forcing and the resulting circulation is predominantly horizontal, bearing strong resemblance to that of quasigeostrophic turbulent flows. The circulation is indeed quasigeostrophic on average in both runs, featuring small $\langle R o\rangle$, negligible contribution of divergence and predominance of horizontal kinetic energy component over the vertical one. In the vortex cores and inside intense filaments, however, dynamics is locally ageostrophic and the strength of the ageostrophic component increases with the strength of the stratification.

Wunsch and Ferrari (2004) suggest that the wind driven near-inertial energy could sustain the small-scale mixing in the deep interior, needed to resupply the available potential energy removed by the overturning and mesoscale eddy generation. Theoretical considerations as well as modelling studies suggest that mesoscale flows could be responsible for transferring wind energy into the ocean interior through polarization of near inertial oscillations (Kunze, 1985; Klein et al., 2004; Danioux et al., 2008). Here we have shown that mesoscale anticyclones are "islands" of increased penetration of wind energy into the ocean interior, representing the maxima of available potential energy. The APE, in turn, is a function of stratification and increases with it. Moreover, we have demonstrated how flows dominated by mesoscale anticyclonic vortices, through trapping and interactions with the internal wave field, can transmit the wind energy to the deeper layers and increase the mixing at depth. We have also found that not only near-inertial oscillations, but also subinertial vortex Rossby Waves contribute to the eddy "chimney effect" (Lee and Niiler, 1998).

In our simulations these physical mechanisms appear to be significantly affected by stratification. We find that the vertical transfer of wind momentum is more pronounced close to the surface when the stratification is stronger and that penetration below the first hundred meters is limited to sporadic events. Differences linked to stratification are apparent when evaluating the vertical velocities and the associated mixing. For the strongly stratified case, the vortex Rossby Waves and 
their interactions with the Ekman circulation induce a more vigorous and spatially-complex $w$ field near the ocean surface that drives a vertical transfer of momentum and tracers, but VRWs are weaker and contain less energy than WS below the first $150 \mathrm{~m}$. For weaker stratification, vertical mixing close to the surface is less intense but below $\sim 150 \mathrm{~m}$ higher values than SS due to a largely increased contribution of both VRWs, whose time scale is on the order of few days, and of near-inertial motions, on a time scale of few hours.

In a turbulent flow dominated by the presence of coherent anticyclones, as it may be the ocean interior away from boundaries and strong currents, the overall diffusivity below the first $100 \mathrm{~m}$ tends therefore to increase with decreasing density differences. On time scales much longer than considered here, the intensified transfer of near-inertial momentum at depth could therefore enhance vertical mixing and potentially weaken further the stratification in a positive feedback loop. More work is needed to test this possibility in realistic configurations, but the implications for climate change scenarios could be severe.

By analyzing results from different general ocean circulation models, Brown and Fedorov (2008) have found a tendency for decreasing trends in both wind power and APE in the tropical Pacific Ocean over the last 50 years. In such a case the weakening of the winds and the decrease in APE could lead to an increase of the vertical mixing due to the turbulent mesoscale flows and further decrease in the thermocline slope.

This work was originally motivated by the idea that the ocean circulation in the Eocene could have been dominated by eddies in presence of a very weak thermocline Hay et al. (2004). We wanted to explore, in a idealized and simple configuration, how the vertical transport within eddies would be affected by stratification. We find that internal wave motion (VRWs and near-inertial contributions) could play an important role in the maintenance of a weak thermocline, through physical mechanisms quite different from what hypothesized by simple quasigeostrophic arguments.

As a note of caution, the reader should be reminded that in our model the vertical mixing coefficient, $K v$, depends on forcing, stratification and velocity field, and is calculated by the KPP scheme (Large et al., 1994). As a result of the interplay between the local stratification properties and the eddies, surface values of $K v$ are very large in the vicinity of the anticyclonic vortices, enhancing their role in the vertical transfer of momentum and tracers into the deeper layers. The results presented may depend on the parameterization scheme. However, the KPP scheme is regarded as a reliable representation of the physics of the vertical mixing in ocean models and is widely implemented (e.g., Li et al., 2001).

In view of the present results on the role of wind-forced mesoscale vortices in the transmission of the wind energy into the ocean and vertical transport, we raise a question whether the ocean circulation models, neither properly resolving mesoscale- and submesoscale dynamics, nor providing for spatial variability in the mixing, have any relevance to the study of the ocean circulation under different climates.

Acknowledgements. We thank Debbie Thomas who inspired this work while visiting Georgia Tech. We also wish to thank Claudia Pasquero and an anonymous reviewer for their valuable comments and suggestions that helped improving the manuscript. AB is supported by NSF OCE-0928495.

Edited by: C. López

Reviewed by: C. Pasquero and another anonymous referee

\section{References}

Arai, M. and Yamagata, T.: Asymmetric evolution of eddies in rotation shallow water, Chaos, 4, 163-173, 1994.

Arbic, B. K., Flierl, G. R., and Scott, R. B.: Cascade inequalities for forced-dissipated geostrophic turbulence, J. Phys. Oceanogr., 37, 1470-2177, 2007.

Babiano, A. and Provenzale, A.: Coherent vortices and tracer cascades in two-dimensional turbulence, J. Fluid Mech., 574, 429_ 448, 2007.

Benitez-Nelson, C. R., Bidigare, R. R., Dickey, T. D., Landry, M. R., Leonard, C. L., Brown, S. L., Nencioli, F., Rii, Y. M., Maiti, K., Becker, J. W., Bibby, T. S., Black, W., Cai, W.-J., Carlson, C. A., Chen, F., Kuwahara, V. S., Mahaffey, C., McAndrew, P. M., Quay, P. D., Rappe, M. S., Selph, K. E., Simmons, M. P., and Yang, E. J.: Mesoscale Eddies Drive Increased Silica Export in the Subtropical Pacific Ocean, Science, 316, 1017-1021, 2007.

Bracco, A. and Pedlosky, J.: Vortex generation by topography in locally unstable baroclinic flows, J. Phys. Oceanogr., 33, 207 219, 2003.

Bracco, A., von Hardenberg, J., Provenzale, A., Weiss, J. B., and McWilliams, J. C.: Dispersion and mixing in quasigeostrophic turbulence, Phys. Rev. Lett., 92(9), 084501, 1-4, 2004.

Brown, J. N. and Fedorov, A. V.: Mean energy balance in the tropical Pacific Ocean, J. Mar. Res., 66(1), 1-23, 2008.

Cho, J. and Polvani, L. M.: The emergence of jets and vortices in freely evolving, shallow- water turbulence on a sphere, Phys. Fluids, 8, 1531-1552, 1996.

Danioux, E. and Klein, P.: A resonance mechanism leading to windforced motions with a $2 \mathrm{f}$ frequency, J. Phys. Ocean., 38, 23222329, 2008.

Danioux, E., Klein, P., and Riviere, P.: Propagation of wind energy into the Deep ocean through a fully turbulent mesoscale eddy field, J. Phys. Oceanogr., 38, 2224-2241, 2008.

Gent, P. R. and McWilliams, J. C.: Isopycnal mixing in ocean circulation models, J. Phys. Ocean., 20, 150-155, 1995.

Graves, L. P., McWilliams, J. C., and Montgomery, M. T.: Vortex evolution due to straining: a mechanism for dominance of strong, interior anticyclones, Geophys. Astro. Fluid, 100(3), 151-183, 2006.

Griffies, S. M.: Development in ocean climate modelling, Ocean Model., 2, 123-192, 2000.

Hay, W. W., Flögel, S., and Söding, E.: Is the initiation of glaciation on Antartica related to a change in the structure of the ocean?, Global Planet. Change, 45, 23-33, 2004. 
Klein, P., Lapeyre, G., and Large, W. G.: Wind ringing of the ocean in presence of mesoscale eddies, Geophys. Res. Lett., 31, L15306, doi:10.1029/2004GL020274, 2004.

Koszalka, I., Bracco, A., McWilliams, J. C., and Provenzale, A.: Dynamics of wind-forced coherent anticyclones in open ocean, $\mathrm{J}$. Geophys. Res., 56, C08011, doi:10.1029/2009JC005388, 2009.

Kunze, E.: Near-inertial wave propagation in geostrophic shear, J. Phys. Oceanogr., 15, 544-565, 1985.

Large, W. G., McWilliams, J. C., and Doney, S.: Oceanic vertical mixing: a review and a model with a non-local K-profile boundary layer parameterization, Rev. Geophys., 32(4), 363403, 1994.

Larichev, V. D. and McWilliams, J. C.: Weakly decaying turbulence in an equivalent-barotropic fluid, Phys. Fluids, 3(5), 938-950, 1991.

Lee, D.-K. and Niiler, P.: The inertial chimney: the near inertial energy drainage from the ocean surface to the deep layers, J. Geophys. Res., 103, 7579-7591, 1998.

Li, X., Chao, Y., McWilliams, J. C., and Fu, L.-L.: A comparison of two vertical-mixing schemes in a Pacific Ocean general circulation model, J. Climate, 14, 1377-1398, 2001.

McGillicuddy, D. J., Anderson, L. A., Bates, N. R., Bibby, T., Buesseler, K. O., Carlson, C. A., Davis, C. S., Ewart, C., Falkowski, P. G., Goldhwait, S. A., Hansell, D. A., Jenkins, W. J., Johnson, R., Kosnyrev, V. K., Ledwell, J. R., Li, Q. P., Siegel, D. A., and Steinberg, D. K.: Eddy/wind interactions stimulate extraordinary mid-ocean plankton blooms, Science, 316, 1021-1026, 2007.

McWilliams, J. C. and Weiss, J. B.: Anisotropic geophysical vortices, Chaos, 4(2), 305-311, 1994.

Oort, A. H., Ascher, S. C., Levitus, S., and Peixoto, J. P.: New estimates of available potential energy in the world ocean, J. Geophys. Res., 94, 3187-3200, 1989.

Pasquero, C., Bracco, A., and Provenzale, A.: Coherent vortices, Lagrangian particles and the marine ecosystem, in: Shallow Flows, edited by: Jirka, G. H. and Uijttewaal, W. S. J., A. A. Balkema Publishers, 339-412, 2004.

Pasquero, C., Bracco, A., Provenzale, A., and Weiss, J. B.: Particle motion in the sea of eddies, in: Lagrangian Analysis and Prediction of Coastal and Ocean Dynamics, edited by: Griffa, A., Kirwan, A. D., Mariano, A. J., Ozgökmen, T., and Rossby, T., Cambridge University Press, chapter 4, 89-118, 2007.
Petersen, M. R., Julien, K., and Weiss, J. B.: Vortex cores, strain cells, and filaments in quasigeostrophic turbulence, Phys. Fluids, 18, 026601, doi:10.1063/1.2166452, 2006.

Polvani, L. M., McWilliams, J. C., Spall, M. A., and Ford, R.: The coherent structures of shallow-water turbulence: deformation radius effects, cyclon/anticyclone asymmetry and gravitywave generation, Chaos, 4(2), 177-186, 1994.

Provenzale, A.: Transport by coherent barotropic vortices, Annu. Rev. Fluid Mech., 31, 55-93, 1999.

Shchepetkin, A. and McWilliams, J. C.: The regional oceanic modelling system (ROMS): a split-explicit, free-surface, topographyfollowing-coordinate oceanic model, Ocean Dynam., 9, 3473404, 2005.

Smith, K. S. and Vallis, G. K.: The scales and equilibration of midocean eddies: freely evolving flow, J. Phys. Oceanogr., 31, 554571, 2001.

Smith, K. S. and Vallis, G. K.: The scales and equilibration of midocean eddies: Forced-Dissipative flow, J. Phys. Oceanogr., 32, 1699-1720, 2002.

Spall, M. A., Pickart, R. S., Fratantoni, P. S., and Plueddeman, A. J.: Western Arctic Shelfbreak eddies: formation and transport, J. Phys. Ocean., 38(8), 1644-1668, 2007.

Stammer, D.: Global characteristics of ocean variability estimated from regional TOPEX/POSEIDON altimeter measurements, J. Phys. Ocean., 27, 1743-1769, 1997.

Vallis, G. K.: Atmospheric and Oceanic Fluid Dynamics: Fundamentals and Large-scale Circulation, Cambridge University Press, New York, US, 2006.

Weiss, J.: The dynamics of enstrophy transfer in two-dimensional turbulence, Physica D, 48, 273-294, 1981.

Wunsch, C. and Ferrari, R.: Vertical mixing, energy and the general circulation of the oceans, Annu. Rev. Fluid Mech., 36, 281-314, 2004.

Yavneh, I., Shchepetkin, A. F., McWilliams, J. C., and Graves, L. P.: Multigrid solutions of rotating, stably stratified flows: the balance equations and their turbulent dynamics, J. Comp. Phys., 136, 245-262, 1997. 